\title{
Statistical Models of Criminal Behavior: The Effects of Law Enforcement Actions
}

\author{
Paul A. Jones ${ }^{1}$, P. Jeffrey Brantingham ${ }^{2}$ and Lincoln R. Chayes ${ }^{1}$
}

September 11, 2009

\author{
${ }^{1}$ Department of Mathematics, UCLA, Los Angeles, CA 90059-1555 USA \\ ${ }^{2}$ Department of Anthropology, UCLA, Los Angeles, CA 90059-1553 USA
}

\begin{abstract}
We extend an agent-based model of crime-pattern formation initiated in Short et al. by incorporating the effects of law enforcement agents. We investigate the effect that these agents have on the spatial distribution and overall level of criminal activity in a simulated urban setting. Our focus is on a two-dimensional lattice model of residential burglaries, where each site (target) is characterized by a dynamic attractiveness to burglary and where criminal and law enforcement agents are represented by random walkers. The dynamics of the criminal agents and the target-attractiveness field are, with certain modifications, as described in Short et al. Here the dynamics of enforcement agents are affected by the attractiveness field via a biasing of the walk, the detailed rules of which define a deployment strategy. We observe that law enforcement agents, if properly deployed, will in fact reduce the total amount of crime, but their relative effectiveness depends on the number of agents deployed, the deployment strategy used, and spatial distribution of criminal activity. For certain policing strategies, continuum PDE models can be derived from the discrete systems. The continuum models are qualitatively similar to the discrete systems at large system sizes.
\end{abstract}

\section{Introduction}

In a previous work 42 a model of criminal behavior was introduced, the purpose of which was to describe, at the statistical level, evolving patterns of criminal activity. The essential components of the model are (a) itinerant criminal agents and (b) target attractiveness. An important feature of the model is that the attractiveness is dynamically updated in response to the history of activity at the target. This in turn has led - at least within the context of the model - to the successful prediction of crime hotspots. The purpose of the current work is to introduce and study the effects of a third (essential) component in the model, namely the presence of law enforcement agents.

We remark that, in the context of this and the earlier work, no serious effort is made to calibrate the model to correspond with actual criminal activities on realistic time scales. This is possible, but is the subject of ongoing research. The goal of these studies is to gain a mechanistic understanding 
of the observed phenomena. It is our belief that the observed large-scale, long-time events are a consequence of cooperative behavior among many interacting constituents. Hence, in accord with the understanding of other cooperative phenomena (e.g., phase transitions in condensed matter physics), once the essential features have been encapsulated, the correct phenomenology will be exhibited independent of the finer details. It is our hope that with the general understanding gained concerning the nature of crime hotspots and a general ansatz concerning the nature of police/criminal interactions, it will be possible to suggest strategies for the allocation of police resources to efficiently combat real-world crime.

\subsection{Background}

It is known that crime is not distributed evenly spatially or temporally. Indeed, even at the local level, a burglary at a particular location evidently enhances the prospects of future criminal activity at the same and at nearby locations $[1,25,26,27,28,42$. One explanation for this effect is found in the "broken windows" theory [50] which posits that the disorder that results from criminal activity - graffiti, broken doors and windows - increases the future rate of such activities [29]. In particular, it is not unreasonable to suppose that after a successful burglary the criminal or an associate is more likely to revisit the "easy" target [28, 51]. Moreover, there are mid and long-range correlations: Some neighborhoods are simply "worse" (i.e. enhance and attract criminal activities more) than others [6, 7, 10, 23, 38. However, quite apart from these intrinsic "background" considerations, it appears that the local mechanism has the potential to create hotspots - areas where the burglary rate is substantially higher than that of its surroundings.

With these considerations in mind, let us proceed with an informal description of the model in Short et al. [42]; a more precise description will be provided in the next subsection. The broken windows effect is reflected in a dynamical property of the targets: associated with each target (house) there is a number which represents the "attractiveness" of the location for burglary by criminal agents. This attractiveness plays two roles: (i) it biases movement of the agents toward targets of higher attractiveness and (ii) determines the rate at which (or probability that) a target is burglarized given that a criminal agent is at the target location. Moreover, the value itself represents the sum of two terms. One component is static and is derived from the intrinsic properties of the house and its neighborhood [2, 12, 21, 35, 39, 40]. The other component is dynamic and is related to local burglary events. How the dynamical component evolves in time will be described shortly.

Thus, effectively, a criminal agent at a particular location has a choice: The agent can either commit a burglary, the probability of which increases with the attractiveness value, or the agent "chooses" to move to a neighboring house. If burglary has been chosen, then the agent is removed from the model. This represents the criminal returning home with the illicit acquisitions. On the other hand, if the criminal agent chooses to move, a neighboring location is selected with a bias favoring neighbors with greater attractiveness.

Next, let us briefly discuss how criminals enter and leave the system. As mentioned above, after a criminal agent has committed a burglary the agent is removed from the system. Criminals can 
also be randomly removed from the system. Indeed since most (low profile) house burglaries are committed by criminal agents who live relatively close to their target [11, 39, 5, 6, 43], it seems reasonable to incorporate a mechanism that limits how far criminals wander from their starting point. To this end, a rate of removal from the system is introduced which may, in the above vernacular, be regarded as a third choice: With some relatively low probability, the agent is simply removed from the environment without having committed a burglary. This probability represents how long, on average, a criminal agent is willing to wander before giving up and returning home. Finally, to model the agents starting from their homes and commencing their searches for a suitable target, criminals are introduced at each site with a given rate.

It is evident that the behavior of criminal agents is strongly tied to the attractiveness values in their local environments. The key feature of the model is a feedback mechanism whereby the converse is also true: local attractiveness values are increased by criminal activities. Specifically, in the event that a house is burglarized, the dynamic attractiveness of the targeted house is increased by a fixed amount [27, 28,.1] This does not represent a permanent change, nor is this the only mechanism for increase of attractiveness. First, the dynamic attractiveness of each house decays in time; thus, if not subjected to further criminal activity, the total attractiveness value of a target returns to its baseline value. Second, the dynamic attractiveness is spatially diffusive: local values are adjusted in accord with the average among neighboring houses.

In the present work, police agents will be introduced into the above model; a schematic overview of the entire setup is provided in Figure 1. Police agents will be permitted to explore the urban environment according to various strategies (for general background see [34, 37, 48]) the central purpose of this work is to investigate different strategies in order to ascertain their relative effectiveness at reducing criminal activity. To accomplish this, we must first decide how to model the interaction of police agents with the criminal agents and/or the urban environment. The vast majority of burglaries go unsolved [52] and, in any case, on-the-spot arrests of burglars are rare. Thus no attempt will be made to model apprehension of criminals by law enforcement agents. Instead, we will aim to incorporate the deterrent effect of police into the system.

Deterrent effects will be modeled in two ways, the first of which impacts criminals directly and the second via a short-term effect of police agents on the urban environment. In general, on the basis of obvious considerations, criminals will avoid committing crimes in the presence of law enforcement. See discussion in [17]. Thus one reasonable mode of interaction is that on encounter with a police agent, a criminal agent might - prematurely - decide to return home. The second mode alters the environment: proximity of police will tend to make the local environment less attractive to the criminal elements. Hence by reducing the attractiveness value in accord with the

\footnotetext{
${ }^{1}$ It is important to note that, in the context of the model, not all criminal actions need be interpreted as actual burglary events. Thus the so called "burglary events" - standing notation to which we will adhere - can represent any criminal activity that increases the perceived attractiveness of the target. Crimes, such as attempted burglaries and vandalism, that are committed on a property may indeed raise that house's attractiveness to criminal elements. Further, see other low level criminal activities done in the neighboring environment, such as a hole cut in a fence to facilitate a getaway, will also increase the rate of future burglaries 29 .
} 
presence of police agents there will be a diminished rate of criminal activity and a tendency for criminals to move away from regions with a high concentration of police agents. It is remarked that both modes are enforcing the same tendencies. Due to limitations of our own resources, we have chosen to focus our attentions on the second option.

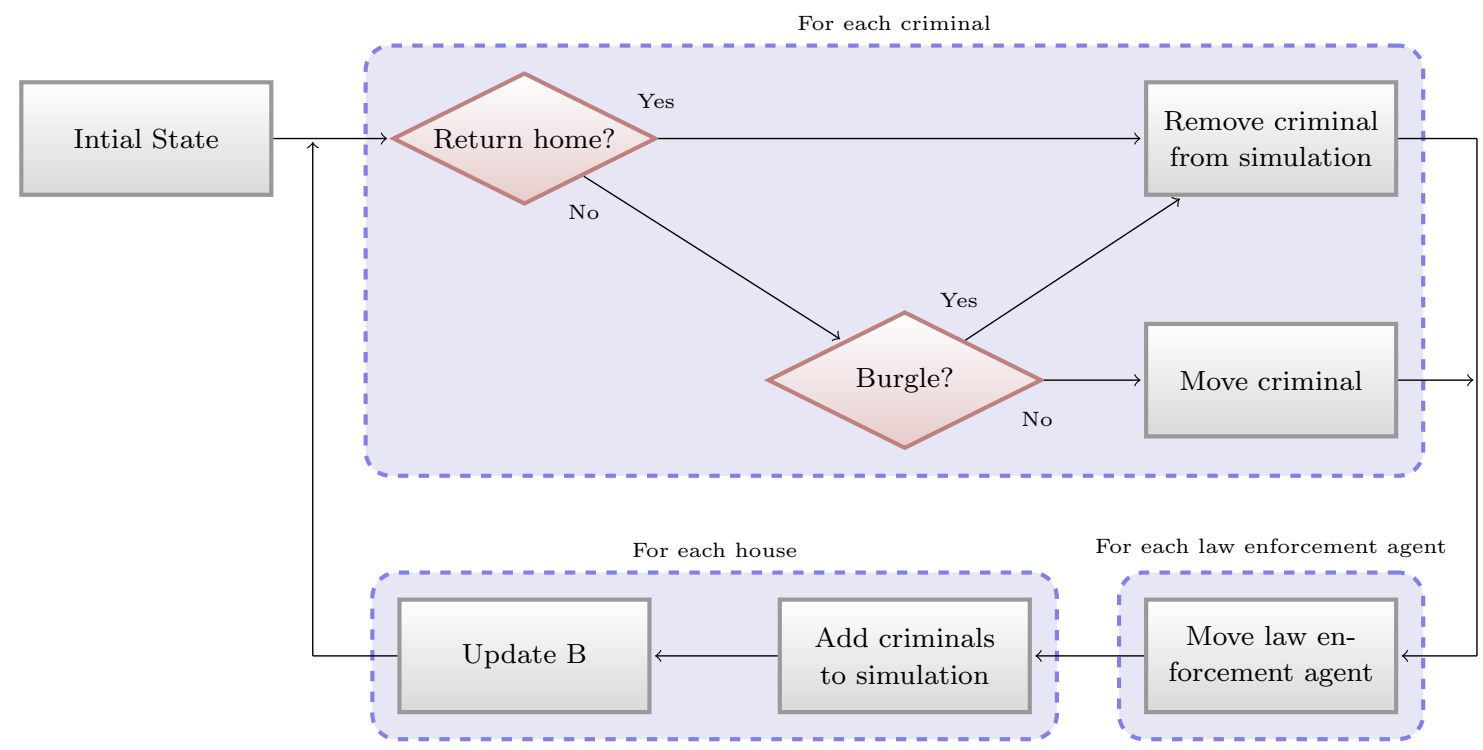

Figure 1: Flowchart summarizing the discrete simulation

\subsection{Formulation of the Model}

We shall now give a more precise description of the model without police agents. The model can be defined on any graph $\mathbb{G}$ (the geometry of which can closely reflect that of an actual city). However for simplicity, we shall place the target houses at points on the $2 \mathrm{D}$ square lattice $\mathbb{Z}^{2}$. We denote a generic position (site) by $s=(i, j)$ with, e.g. $0 \leq i \leq M, 0 \leq j \leq N$. At each site $s$, the number $A_{s}(t)$ represents the attractiveness of the target at $s$ at the integer time $t$. As noted in the previous subsection, attractiveness is the sum of two components, $A_{s}(t)=A_{s}^{0}+B_{s}(t)$, where $A_{s}^{0}$ is the time-independent component and, $B_{s}(t)$, evolves dynamically.

The evolution in time of $B_{s}(t)$ is driven by burglary events near $s$. The probability that a criminal agent at site $s$ will choose to commit a burglary is

$$
p_{s}(t)=\frac{\epsilon A_{s}(t)}{1+\epsilon A_{s}(t)}
$$

where $\epsilon>0$ determines the effectiveness of $A_{s}$ in this context.2 The increase in the dynamic

\footnotetext{
${ }^{2}$ The equation 1.1 differs somewhat from its counterpart in 42 since, in that context, the model was driven by rates rather than probabilities. Although there is a technical distinction between the two approaches, we do not
} 
attractiveness at site $s$ that results from $E_{s}(t)$, the number of burglary events at time $t$, is:

$$
B_{s}(t+1)=B_{s}(t)+\theta E_{s}(t)
$$

where $\theta$ is the increase in attractiveness from a single burglary.

Now the decay of attractiveness over a time step ${ }^{3}$ is

$$
B_{s}(t+1)=(1-\omega) B_{s}(t)
$$

where $\omega$, the rate of decay, is a constant between 0 and 1 . Finally, the diffusion of attractiveness is modeled by

$$
B_{s}(t+1)=(1-\eta) B_{s}(t)+\frac{\eta}{4} \sum_{s^{\prime} \sim s} B_{s^{\prime}}(t)
$$

where $\eta$, the rate of diffusion, is a constant between 0 and 1 and $s^{\prime} \sim s$ denotes $s^{\prime}$ is a neighbor of $s$ - that is $\left\|s^{\prime}-s\right\|=1$. Equation $(1.2)$ can be rewritten as

$$
B_{s}(t+1)=B_{s}(t)+\frac{\eta}{4} \Delta B_{s}(t)
$$

where $\Delta$ is the discrete Laplacian

$$
\Delta B_{s}(t)=\sum_{s^{\prime} \sim s} B_{s^{\prime}}(t)-4 B_{s}(t)
$$

Thus, if $n_{s}(t)$ denotes the number of criminal agents at the site $s$ at time $t$, we may combine all of the above to obtain:

$$
B_{s}(t+1)=\left[B_{s}(t)+\frac{\eta}{4} \Delta B_{s}(t)\right](1-\omega)+\theta p_{s}(t) n_{s}(t)
$$

Remark 1. We remark that the equation 1.3 is in actuality a formal identity since, strictly speaking, the right hand side is the average value of $B_{s}$ at time $t+1$ given the (random) values of the various other quantities at time $t$. It is not possible to fully justify equation (1.3) by taking the average of the right side since the random variables $n_{s}$ and $p_{s}$ are not necessarily independent. While, from a mathematical perspective, we consider these points to be important, we do not anticipate a significant impact on the large-scale, long-time behavior of the system to result from the neglect of these considerations. This anticipation has been born out by the close similarities between the discrete agent based models (which, necessarily, includes correlations) and the naïve continuum limiting PDE in which these effects have been neglected. Approximations of this sort are not without precedent: In particular, the philosophy, as discussed in Spohn [44], is that the important (large-scale) correlations are reflected in the intrinsic non-linearities of the continuum

regard such differences as important. Indeed in the naïve continuum limit, the denominator washes out and thus coincides with the version of equation (1.1) according to rates.

${ }^{3}$ For present purposes, a single time step is the time scale in which each agent or target is typically updated once. In the vernacular of spin-systems, this is one Monte Carlo step per spin. 
limit. In all examples of similar models that have been mathematically analyzed (not to mention a plethora of systems that have received attention in the physics community), the appropriate continuum limit turns out to be essentially that which is obtained by the neglect of local correlations. However, it is not necessarily the case that the continuum parameters are exactly the parameters obtained by the so called naïve scaling. Notwithstanding, in the context of the present work we shall adhere to this ideology and will consider the continuum systems obtained by naive scaling with neglect of correlations.

In the event that a criminal agent does not commit a burglary, the agent will be removed or moved to a neighboring site. In the latter case, the site will be chosen randomly, but biased in the direction of high attractiveness. Here the probability a criminal agent will move from site $s$ to a neighbor $r$ is given by

$$
\frac{A_{r}(t)}{\sum_{s^{\prime} \sim s} A_{S^{\prime}}(t)}
$$

Given the number of criminals, $n_{s}(t)$ at site $s$, let us now calculate the number that will be at the site at time $t+1$. The model, in fact, demands that all of the criminals at site $s$ at time $t$ will be gone by time $t+1$ (criminals agents are allowed one of the following three options: move to another site, commit a burglary - in which case they are removed - or be removed without committing a burglary). Thus the expected number of criminals at site $s$ at time $t+1$ is given by the influx from neighboring sites plus a term accounting for their spontaneous creation:

$$
\rho_{s}(t+1)=(1-\Lambda) A_{s}(t) \sum_{s^{\prime} \sim s} \frac{\left(1-p_{s^{\prime}}(t)\right) \rho_{s^{\prime}}(t)}{T_{s^{\prime}}(t)}+\Gamma .
$$

In the above, $\Lambda$ is the probability that a criminal will be removed without committing a burglary, $\Gamma$ is the probability at a criminal will be added the site (representing a new criminal "starting from home") and $T_{s}(t)=\sum_{s^{\prime} \sim s} A_{s^{\prime}}(t)$.

This describes the discrete model. In Short et al. [42] the "naïve" continuum limit (c.f. above remark) for the above system was derived using a common procedure in mathematical biology $[3,4,15,16,20,21,24,32,35,36,45]$ :

$$
\begin{gathered}
\frac{\partial B(\mathbf{x}, t)}{\partial t}=\frac{\eta}{4} \Delta B(\mathbf{x}, t)-\omega B(\mathbf{x}, t)+\theta \rho(\mathbf{x}, t) \epsilon A(\mathbf{x}, t) \\
\frac{\partial \rho(\mathbf{x}, t)}{\partial t}=-\epsilon A(\mathbf{x}, t) \rho(\mathbf{x}, t)-\Lambda \rho(\mathbf{x}, t)+\Gamma+\frac{1}{4} \Delta \rho(\mathbf{x}, t)-\frac{1}{2} \nabla \cdot(\rho(\mathbf{x}, t) \nabla \log A(\mathbf{x}, t)) .
\end{gathered}
$$

These resulting equations are closely related to the Keller-Segel model for chemotaxis [13, 14, 19, 22, 26, 30, 33, 41, 46, 47.

As the model evolves in time it is noticed that, dependent on parameters and initial conditions, only three distinct types of behaviors of the attractiveness field appear to be possible [42]; output from computer simulations can be found in Figure 2. The simplest is spatial homogeneity (Figure 2I) with an attractiveness field that is essentially the same everywhere. The next possible behavior 
is dynamic hotspots (not shown). In this regime, hotspots - regions of large value of $A$ and/or high rate of burglary - appear and disappear as time evolves. Hotspots may reappear in the same places repeatedly, or they may appear in seemingly random locations. However, it seems that this sort of behavior is a result of random noise augmented by the finite size of the system. In particular, no such behavior is observed in the continuum PDE and in the discrete model, noisy/dynamic behavior does not persist in large scale systems. This does not imply that the effect is unimportant or irrelevant to actual urban activities. Indeed, it is worth remarking that the latter is more accurately modeled by a discrete system than the continuum limit thereof. The third and final regime is that of stationary hotspots (Figures $2 \mathrm{I}-\mathrm{III}$ ). This regime is described by hotspots that nucleate, grow to a characteristic size, and then remain stationary "for as long as one cares to observe"; see [18]. Here the system is apparently exhibiting one of many possible long-lived steady states.

To perform any meaningful analysis on our simulations, we need a metric to determine the amount of criminal activity. Ostensibly, we could use the number of burglary events, but this has a few drawbacks. First, there are families of systems with the same behavior, but different levels of criminal activity for example, the crime patterns in two instances may be the same, but the number of criminals vary - an effect that can be achieved by reducing $\theta$ and increasing $\Gamma$. Moreover it is possible to produce two systems of different sizes with the same crime patterns. Second, burglary is a relativity rare event; the number of burglaries that occur in any given time-step may vary widely. Thus, to draw any meaningful information from the number of burglaries, a temporal average must be taken.

The spatial average of $B_{s}(t)$, which we denote $B_{\mathrm{Av}}(t)$, is related to the amount of criminal activity. For any time, $T$,

$$
B_{\mathrm{Av}}(T)=(1-\omega)^{T} B_{\mathrm{Av}}(0)+\theta \sum_{n=1}^{T}(1-\omega)^{n-1} E_{\mathrm{Av}}(T-n+1)
$$

where $E_{\mathrm{Av}}(t)$ is the spatial average of number of burglaries that occurred at each site at time $t$. As $0<\omega<1, B_{\mathrm{Av}}(t)$ provides a reasonable proxy for amount of criminal activity, while averaging out some of the volatility. Thus in what follows, we will we equate $B_{\mathrm{Av}}(t)$ to the level of criminal activity.

This leads to natural question: how much crime do we expect? To answer we exploit the relationship between the discrete and continuum models. Following the procedure used to calculate equations (3.4) and (3.5) we estimate the attractiveness and criminal density of spatially homogeneous steady state solutions

$$
\begin{aligned}
\bar{B} & \equiv \frac{\epsilon \omega \overline{A^{0}}+\epsilon \theta \Gamma-\omega \Lambda+\sqrt{\left(\epsilon \omega \overline{A^{0}}+\epsilon \theta \Gamma-\omega \Lambda\right)^{2}+4 \epsilon \omega^{2} \overline{A^{0}} \Lambda}}{2 \epsilon \omega}-\overline{A^{0}} \quad \text { and } \\
\bar{\rho} & \equiv \frac{\epsilon \omega \overline{A^{0}}+\epsilon \theta \Gamma+\omega \Lambda-\sqrt{\left(\epsilon \omega \overline{A^{0}}+\epsilon \theta \Gamma+\omega \Lambda\right)^{2}-4 \epsilon \omega \Gamma \Lambda \theta}}{2 \epsilon \Lambda \theta}
\end{aligned}
$$


where $\overline{A^{0}}$ is the spatial average of $A_{s}^{0}$. Strictly speaking, these equations are only valid for the continuum model, but for large enough system sizes the two models behave similarly. Thus, the above quantities in equations (1.7)-(1.8) provide a reasonable estimate for a steady state solution with no hotspots.

When crime hotspots are present, the estimates provided by equations $1.7-1.8$ are no longer valid. Figure 2 shows that we can expect much higher levels of criminal activity when hotspots are present.

\section{Law Enforcement}

In this section we add law enforcement agents to the above model with the goal of reducing the amount of criminal activity in the system. In the simulations and the linear stability analysis presented by Short et al. [42], it is clear that the presence or absence of hotspots is a threshold phenomenon - small changes in parameters can create a large change in the system. Furthermore, as demonstrated by simulations of the model used in this present work (Figure 2), the presence of hotspots may increase the overall level of criminal activity while the total number of active criminal agents may decline. These two points suggest that a relativity small number of law enforcement agents acting appropriately can have a dramatic effect.

The remainder of this section is divided into three parts. First, in modeling law enforcement agents we must consider their effect on the environment and how criminal agents respond to their presence. We then present three different methods to determine the agents' patrol routes. Finally, we examine the effect of the law enforcement agents in computer simulations. We will observe that the primary factor in the law enforcement agents' effectiveness is their deployment strategy.

\subsection{Modeling the Effects of Law Enforcement}

We add law enforcement agents to the model starting with the lattice version. Let $\kappa_{s}(t)$ be the number of enforcement agents at the site $s$ at time $t$. These agents will only attempt to deter the criminal agents from committing burglary events. Below, we will present two mechanisms: The first modifies how criminal agents perceive their environments. The other has a direct effect on the agents' actions.

2.17 Perception modification In the context of the model, both the movement of a criminal agent and the criminal agent's "decision" to commit a burglary are affected only by the local target attractiveness values. If we allow enforcement agents to modify the attractiveness values, they will ultimately be able to control the level of criminal activity. However, the law enforcement effect must not be permanent; if there is no longer a law enforcement presence, criminal agents will once again find the house attractive to burglary.

With these considerations in mind, we introduce a variable that represents the attractiveness 


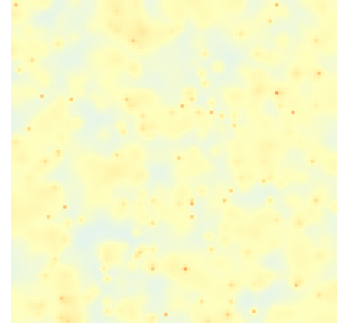
(a) $t=1 \times 10^{3}$
$\mathrm{ACA}=169$
$B_{\mathrm{Av}}=0.598$
(b) $t=32 \times 10^{3}$
$\mathrm{ACA}=180$
$B_{\mathrm{Av}}=0.596$

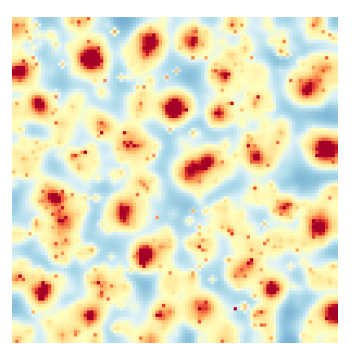

(a) $t=1 \times 10^{3}$

$\mathrm{ACA}=2310$

$B_{\mathrm{Av}}=0.525$

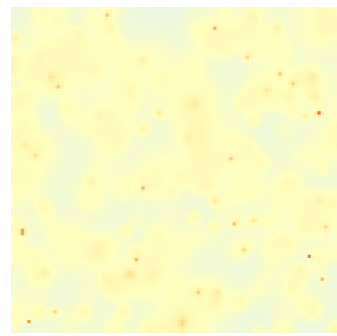
(a) $t=1 \times 10^{3}$
$\mathrm{ACA}=2891$
$B_{\mathrm{Av}}=0.359$
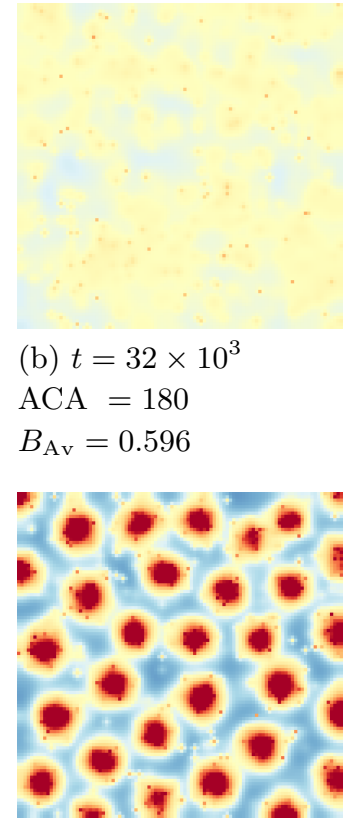

(b) $t=32 \times 10^{3}$

$\mathrm{ACA}=1903$

$B_{\mathrm{Av}}=0.732$

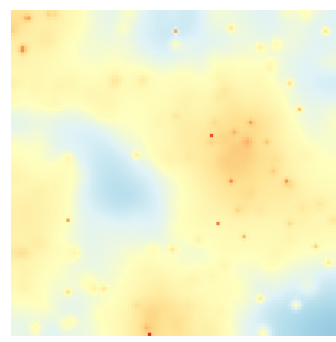

(b) $t=32 \times 10^{3}$

$\mathrm{ACA}=2761$

$B_{\mathrm{Av}}=0.396$ (c) $t=128 \times 10^{3}$

$\mathrm{ACA}=187$

$B_{\mathrm{Av}}=0.604$

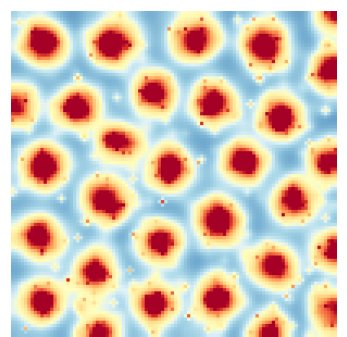

(c) $t=128 \times 10^{3}$

$\mathrm{ACA}=1755$

$B_{\mathrm{Av}}=0.751$

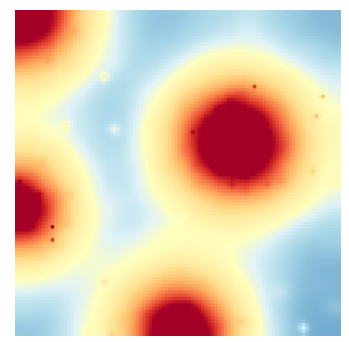

(c) $t=128 \times 10^{3}$

$\mathrm{ACA}=1772$

$B_{\mathrm{Av}}=0.728$
(I) Low Activity. Parameters of the model insufficient to cause hotspots. The system is nearly spatially homogeneous and in particular is close to the predicted value:

$$
\begin{aligned}
B_{\mathrm{Av}}\left(128 \times 10^{3}\right) & =0.604 \\
\text { and } \bar{B} & =0.598 .
\end{aligned}
$$

(II) Small Hotspots. Here the spatial average, $B_{\mathrm{Av}}\left(128 \times 10^{3}\right)=$ 0.751 , is much higher than the predicted homogeneous equilibrium value, $\bar{B}=0.352$. So we see that the formation of hotspots does not just simply redistribute criminal activity, instead it more than doubles the crime rate.

(III) Large Hotspots. Here again we note that the spatial average, which is $B_{\mathrm{Av}}\left(128 \times 10^{3}\right)=0.773$, is much higher than the predicted homogeneous equilibrium value, $\bar{B}=0.352$. Due to the scaling of the parameters, hotspots take longer to form in this simulation.

Figure 2: Simulations showing the formation of hotspots. All simulations were run on a $100 \times 100$ grid with background attractiveness, $A^{0} \equiv A_{s}^{0}=0.1$. We output the results of the simulations as a color map using the predicted average attractiveness, $\bar{A}$, as the midpoint. Values of $A_{s}(t)$ that satisfy $0 \leq A_{s}(t) \leq \bar{A}$ are represented with colors ranging from dark blue to light blue to light yellow. The colors light yellow to orange to dark red represent values of $A_{s}(t)$ between $\bar{A}$ and $5 \bar{A}$. Attractiveness values above $5 \bar{A}$ are also represented with dark red. Each simulation was started with initial conditions $\lfloor 10000 \bar{\rho}\rfloor$ randomly placed criminals and $A_{s}(0)=\bar{A}$. In Ia - Ic, the simulation was run with the parameters $\eta=0.05, \theta=1.0, \omega=4 \times 10^{-4}, \epsilon=0.02, \Gamma=2.5 \times 10^{-4}$, and $\Lambda=6.25 \times 10^{-4}$. In IIa-IIc the simulation was run with parameters $\eta=0.05, \theta=1.0, \omega=3 \times 10^{-3}, \epsilon=8 \times 10^{-3}, \Gamma=4 \times 10^{-3}$, and $\Lambda=4 \times 10^{-3}$. Finally, IIIa-IIIc displays the results of a simulation run with parameters $\eta=0.05, \theta=1.0, \omega=1.875 \times 10^{-4}$, $\epsilon=5 \times 10^{-4}, \Gamma=2.5 \times 10^{-4}$, and $\Lambda=6.25 \times 10^{-4}$. In all of the above, ACA denotes the number of active criminal agents. 
values that criminals perceive in the presence of enforcement agents. Thus

$$
\tilde{A}_{s}(t) \equiv e^{-\chi \kappa_{s}(t)} A_{s}(t)
$$

where $\chi$ is a positive constant.

Now we modify our discrete formulas to include the effect of police officers. The probability that a criminal agent commits a burglary at site $s$ at time $t$ is

$$
\tilde{p}_{s}(t)=\frac{\epsilon \tilde{A}_{s}(t)}{1+\epsilon \tilde{A}_{s}(t)}
$$

The probability that a criminal agent moves from site $s$ to a neighboring site $n$ is

$$
\frac{\tilde{A}_{n}(t)}{\sum_{s^{\prime} \sim s} \tilde{A}_{s^{\prime}}(t)} \text {. }
$$

Therefore equation (1.4) becomes

$$
\rho_{s}(t+1)=(1-\Lambda) \tilde{A}_{s}(t) \sum_{s^{\prime} \sim s} \frac{\left(1-\tilde{p}_{s^{\prime}}(t)\right) \rho_{s^{\prime}}(t)}{\tilde{T}_{s^{\prime}}(t)}+\Gamma,
$$

where

$$
\tilde{T}_{s}(t)=\sum_{s^{\prime} \sim s} \tilde{A}_{s^{\prime}}(t)
$$

Changing the evolution of the criminals, however, also changes the evolution of $B_{s}(t)$. Thus equation (1.3) becomes

$$
B_{s}(t+1)=\left[B_{s}(t)+\frac{\eta}{4} \Delta B_{s}(t)\right](1-\omega)+\theta \frac{\epsilon \tilde{A}_{s}(t)}{1+\epsilon \tilde{A}_{s}(t)} n_{s}(t) .
$$

These give the naïve continuum formulas

$$
\begin{gathered}
\frac{\partial B(\mathbf{x}, t)}{\partial t}=\frac{\eta}{4} \Delta B(\mathbf{x}, t)-\omega B(\mathbf{x}, t)+\theta \rho(\mathbf{x}, t) \epsilon \tilde{A}(\mathbf{x}, t) \quad \text { and } \\
\frac{\partial \rho(\mathbf{x}, t)}{\partial t}=-\epsilon \tilde{A}(\mathbf{x}, t) \rho(\mathbf{x}, t)-\Lambda \rho(\mathbf{x}, t)+\Gamma+\frac{1}{4} \Delta \rho(\mathbf{x}, t)-\frac{1}{2} \nabla \cdot(\rho(\mathbf{x}, t) \nabla \log \tilde{A}(\mathbf{x}, t)) .
\end{gathered}
$$

In short, we have equations $1.5-1.6$ with $A$ replaced by $\tilde{A}$. This is not to say the systems are identical since now equations $(2.3)-(2.4)$ are part of a larger conglomerate in which the dynamics of $\kappa_{s}$ are included.

2.1b Behavior modification There is another possible model for the interaction of cops and criminals. Rather than the cops affecting the environment, they will influence the criminals directly. If a criminal agent comes into contact with an enforcement agent, then with some probability, the criminals will opt to "return home". More precisely, with probability

$$
\frac{J \kappa_{s}(t)}{1+J \kappa_{s}(t)}
$$


a criminal agent at site $s$ will be removed. Here $J$ is a positive constant. Thus equation (1.4) becomes

$$
\rho_{s}(t+1)=(1-\Lambda) A_{s}(t) \sum_{s^{\prime} \sim s} \frac{1}{1+J \kappa_{s^{\prime}}(t)} \frac{\left(1-p_{s^{\prime}}(t)\right) \rho_{s^{\prime}}(t)}{T_{s^{\prime}}(t)}+\Gamma
$$

and the corresponding continuum equation becomes

$$
\frac{\partial \rho(\mathbf{x}, t)}{\partial t}=(-\epsilon A(\mathbf{x}, t)-\Lambda-J \kappa(\mathbf{x}, t)) \rho(\mathbf{x}, t)+\Gamma+\frac{1}{4} \Delta \rho(\mathbf{x}, t)-\frac{1}{2} \nabla \cdot(\rho(\mathbf{x}, t) \nabla \log A(\mathbf{x}, t))
$$

\subsection{Dynamics of Law Enforcement}

As eluded to earlier, the above only covers part of the relationship between law enforcement, criminals, and their environment; the choice of the law enforcement agents' patrol routes will also influence the evolution of the rest of the system. To proceed, we make a couple of simplifying assumptions on the behavior of the enforcement agents: First, we will demand that the number of agents on patrol is constant. This reflects, in part, the reality of the limited resources of the police department. We will also assume that the agents move through the city on the ground (i.e., on foot, car, or some other vehicle). In other words, law enforcement will move through the system, house to house, like the criminal agents. We will propose various strategies that satisfy the above criteria and compare their relative effectiveness.

2.2 Random Walkers One possibility is for law enforcement agents to patrol random routes. Here police do not focus their attention in any particular place. The hope is that criminal activity will be reduced, as the criminal agents will never know when an enforcement agent will be near. This method has been tried, without much success, in Kansas City [31]. We model the patrols by having the law enforcement agents perform an unbiased simple random walk. Thus, the expected number of law enforcement agents at site $s$ and at time $t+1$ is

$$
\kappa_{s}(t+1)=\frac{1}{4} \sum_{s^{\prime} \sim s} \kappa_{s^{\prime}}(t)=\kappa_{s}(t)+\frac{1}{4} \Delta \kappa_{s}(t)
$$

This leads to the continuum equation

$$
\frac{\partial \kappa(\mathbf{x}, t)}{\partial t}=\frac{1}{4} \Delta \kappa(\mathbf{x}, t)
$$

The random patrol method has an obvious downside: enforcement agents will often be located in places where the level of criminal activity is already low. We will now introduce two alternative schemes in which police will concentrate their attention in areas where their presence will have a greater effect.

$2.2 \mathrm{~b}$ Cops on the Dots In this scheme the law enforcement agents move randomly but with a bias in the direction of high attractiveness. We will call this method of policing cops on the dots.

Traditionally, some police departments have marked criminal events with markers or dots on a centrally located map. Police officers are then directed to focus on patrolling the areas denoted 
by the dots. Similarly, in our model the law enforcement agents will tend to patrol areas with relatively high attractiveness - areas correlated with a high number of individual burglary events.

As we will see, cops on the dots is effective in reducing criminal activity. In the model, criminal agents tend to move from areas of low attractiveness to places where it is higher. Furthermore, criminal agents are more likely to commit crimes in these areas of higher attractiveness. Cops on the dots enforces the reverse tendency. Law enforcement agents patrol areas with high attractiveness which reduces the likelihood that criminal agents will commit crimes in these areas (and, in the perception modification model, biases them toward locations with lower attractiveness - places where they are less likely to commit crime). A criminal agent in this situation is more likely to return home without having performed any criminal activity. The overall effect is that the global statistical rate of burglary is lowered.

We model cops on the dots as follows: The probability a law enforcement agent moves from a site $s$ to a neighboring site $d$ is

$$
\frac{A_{d}(t)}{\sum_{s^{\prime} \sim s} A_{s^{\prime}}(t)} \text {. }
$$

We then see that the expected number of law enforcement agents at site $s$ at time $t+1$ is

$$
A_{s}(t) \sum_{s^{\prime} \sim s} \frac{\kappa_{s^{\prime}}(t)}{T_{s^{\prime}}(t)} .
$$

The corresponding continuum equation reads

$$
\frac{\partial \kappa(\mathbf{x}, t)}{\partial t}=\frac{1}{4} \Delta \kappa(\mathbf{x}, t)-\frac{1}{2} \nabla \cdot(\kappa(\mathbf{x}, t) \nabla \log A(\mathbf{x}, t)) .
$$

2.2 Peripheral Interdiction To maximize the deterrent effect given the constraint of limited resources, we introduce another scheme. We will send the law enforcement agents to the perimeters of the hotspots rather than the centers. Since the area of a hotspot grows as a square of its radius, but the perimeter grows only linearly, we expect that this method will be more effective for larger hotspots.

Like cops on the dots, this method reduces criminal activity in areas where the rate is the highest. We have already observed that criminals tend to move to areas with high attractiveness. In fact, this mechanism is largely responsible for the higher rate of criminal activity in these locations. When the law enforcement agents encircle the hotspot they are reducing the rate at which this advection occurs, thereby lowering the rate of criminal activity. We call this method of policing peripheral interdiction.

An artifact of the model is a technical difficulty in biasing the enforcement agents towards the peripheries of the hotspots. Of course in realistic situations, this would be achieved by dispatching under a centralized control. For our purposes, the steps $s \rightarrow s^{\prime}$ of the enforcement agents are biased by weights proportional to $\exp \left\{-\left|c_{1} B_{s^{\prime}}-c_{2}\right|\right\}$ where $c_{1}$ and $c_{2}$ are constants chosen according to the parameters of the simulation. This term biases the agents to a certain level of $B_{s^{\prime}}$; in our case, to a level of attractiveness found on the perimeter of the hotspots. Unfortunately, considering 
the difficulty of these sorts of biases - not to mention the unrealistic assumption of enforcement agents moving autonomously according to such a bias - a continuum PDE analysis of the peripheral interdiction strategy is impractical. In this work, we will be content with the results of the discrete simulations.

\subsection{Results of Discrete Simulations}

In this section we will describe the results of computer simulations of the discrete model outlined in this work. Here, we only present the output using the perception modification model, but we note that both schemes are effective in performing the task that they were created for - namely the reduction of crime.

Regardless of which policing scheme is used we notice some general trends. First, a reduction in criminal activity is correlated with an increase in the number of active criminal agents in our system. While surprising at first, this is to be expected. Criminals are removed from the system when they commit a burglary and reintroduced at a rate that is independent of all other quantities. Thus, if there are fewer burglaries there will be more active criminal agents searching for viable targets. Both aspects of the model though simplified, are consistent with criminological observations. The propensity to offend is widely distributed within populations and the suppression of criminal opportunity, though reducing crime, does not eliminate criminal propensity [25].

We also note that even after law enforcement agents have eliminated a hotspot it may be the case that, nearby, another hotspot will emerge. Indeed, even with law enforcement agents present, a region may be on the verge of instability which means that a hotspot will nucleate with their diminished presence. Thus, under certain circumstances, even after hotspots haver been eliminated, we may expect their reappearance - at least temporarily. This effect will be more noticeable with small hotspots as they tend to form more quickly while the time it takes the law enforcement agents to react and respond to a new hotspot does not depend on its size.

The elimination of crime hotspots may have another unfortunate side effect: while crime is reduced in the areas with the highest criminal activity it may actually be increased in areas where the criminal activity is lower. Displacement of crime has been one outcome observed in field-based tests of hotspot policing [9]. If criminal agents are spending more time outside the center of a crime hotspot, they will be more likely to commit crimes in low crime areas.

Starting with the same law enforcement resources, it is seen that the schemes have vastly different effectiveness (Figures 5 and 6). The worst scheme is random which is largely ineffective in reducing criminal activity (without the commitment of saturation level resources). Conversely, cops on the dots and peripheral interdiction are far better at eliminating criminal activity. Cops on the dots tends to be more effective with small hotspots and peripheral interdiction is more effective with larger hotspots.

Cops on the dots tends to scale smoothly with the number of law enforcement agents. Peripheral

interdiction exhibits more of a threshold effect. When the number of agents is too small to seal off 
the perimeter of even a single hotspot, peripheral interdiction is largely ineffective. However once the number of agents has passed a minimum threshold, peripheral interdiction is suddenly effective. This suggests that under extreme conditions of high criminal activity and limited enforcement resources, cops on the dots is the better scheme.

Finally, we see that cops on the dots seems to eliminate hotspots faster than peripheral interdiction. This is mostly due to the fact that, in the context of the model, there is a delay time for the biased random movements of the enforcement agents to find a hotspot and set up a perimeter. In practice this would be mitigated by a centralized dispatching scheme: By telling enforcement agents exactly where to patrol, an effective perimeter will be set up almost immediately.

We also notice that the hotspots tend to be eliminated sequentially, when peripheral. Once one hotspot has been eliminated, law enforcement agents are free to move to other hotspots, initiating action or helping to seal off their perimeters.

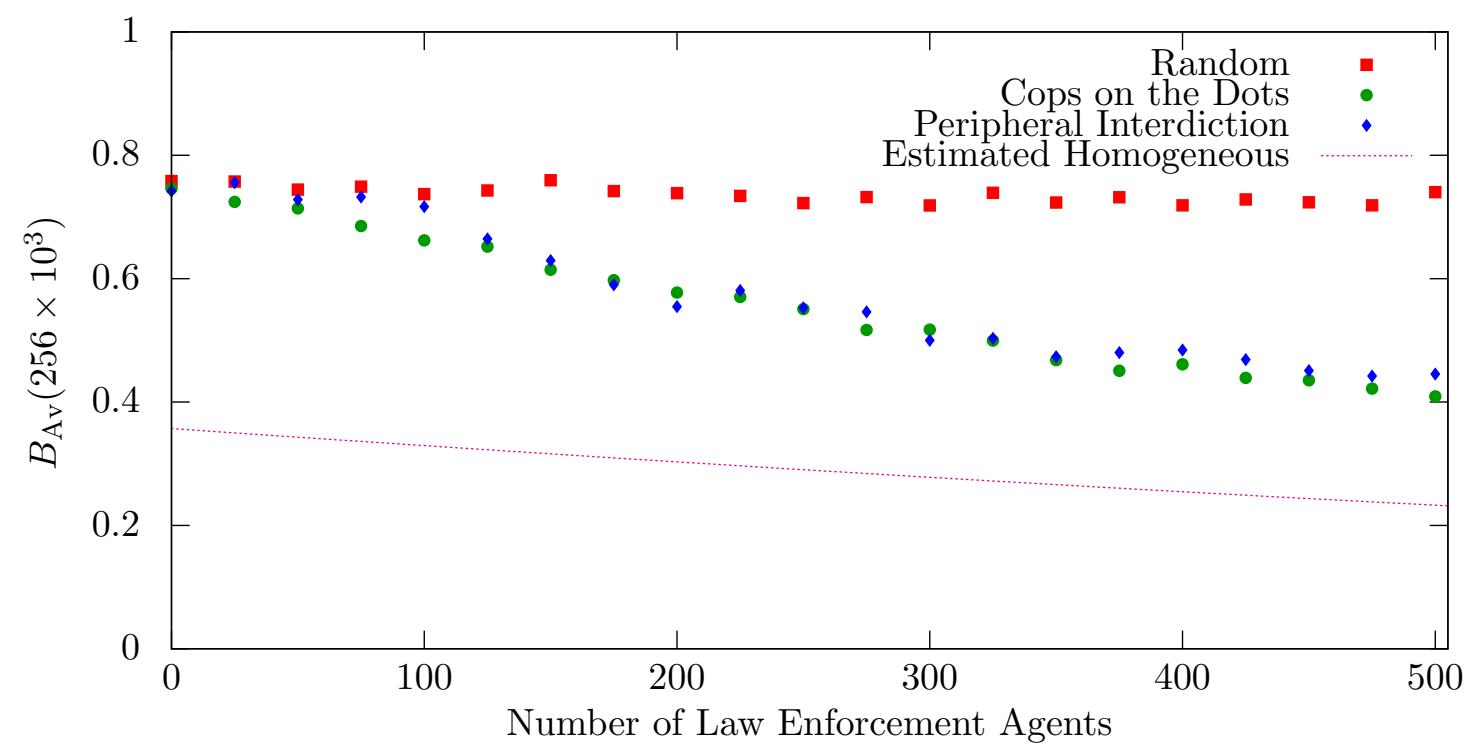

Figure 3: The relative effectiveness of law enforcement schemes with small hotspots. Each simulation is a continuation of the one displayed in Figure 2 II - that is, the parameters are the same and initial conditions are given by the state of the system at Figure 2 IIc $\left(t=128 \times 10^{3}\right)$. The unbiased walkers scheme performs poorly. Peripheral interdiction and cops on the dots are roughly comparable.

\section{Analysis}

In this section we will analyze the continuum equations to determine the effect of law enforcement agents. The first step is to simplify equations 2.3 and 2.4 . If we assume that $A^{0}$ is spatially (and temporally) homogeneous then equation $(2.3)$ can be written in terms of $A$ alone. Furthermore, by various rescalings and redefinitions $\left(\rho^{\star}=\theta \rho, \Gamma^{\star}=\theta \Gamma, \eta^{\star}=\frac{1}{4} \eta\right.$ and $C=\omega A^{0}$ and omitting the $\star^{\prime}$ s $)$ 


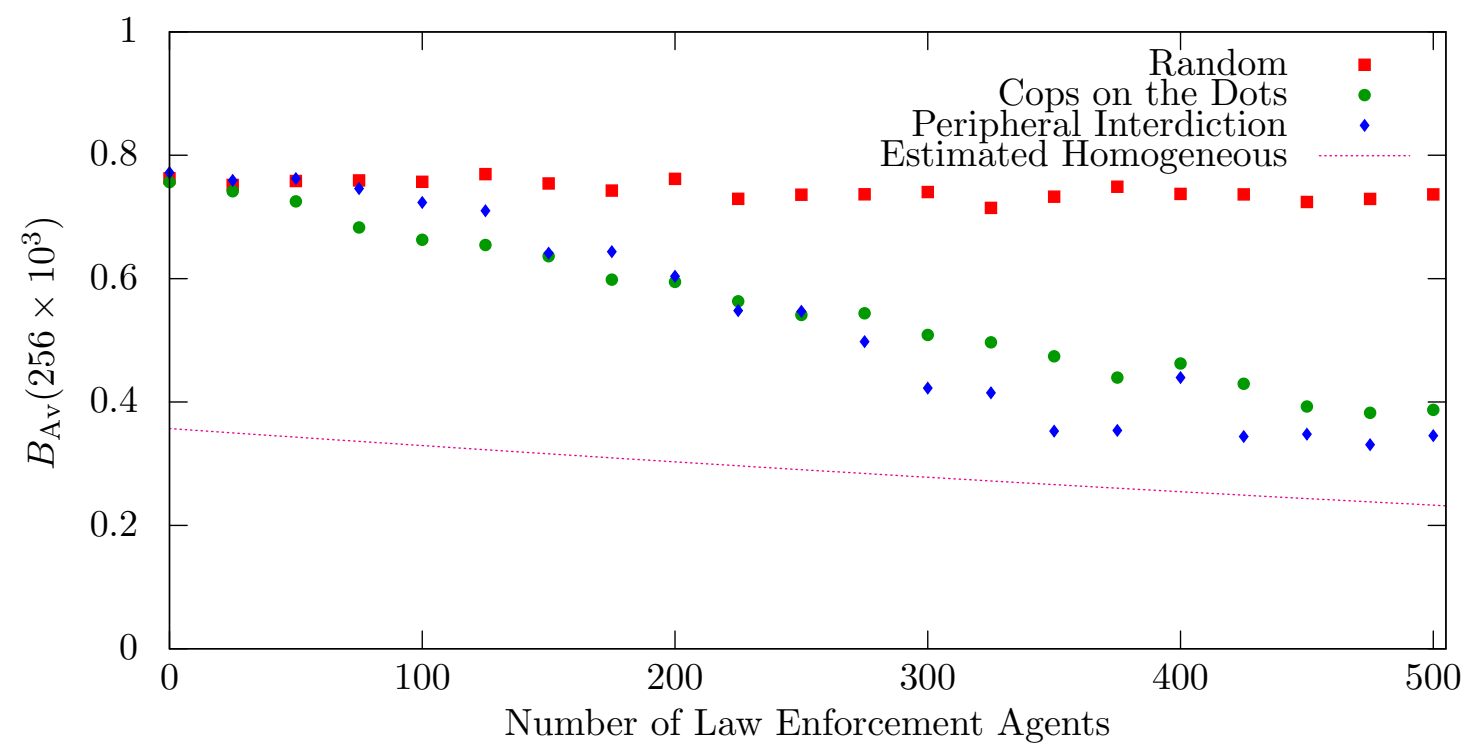

Figure 4: The relative effectiveness of law enforcement schemes with large hotspots. Each simulation is a continuation of the one displayed in Figure 2 III - that is, the parameters are the same and initial conditions are the state of the system at Figure 2 IIIc $\left(t=128 \times 10^{3}\right)$. The unbiased walkers scheme performs poorly. Cops on the dots reduces crime better than peripheral interdiction for low numbers of law enforcement agents. Peripheral interdiction, however, outperforms cops on the dots as the number of law enforcement agents increases.

we arrive at

$$
\begin{aligned}
& \frac{\partial A}{\partial t}=\eta \Delta A-\omega A+C+\epsilon \rho \tilde{A} \\
& \frac{\partial \rho}{\partial t}=\Gamma-\Lambda \rho-\epsilon \rho \tilde{A}+\nabla \cdot(\nabla \rho-2 \rho \nabla \log \tilde{A}) .
\end{aligned}
$$

We begin our study of the system by analyzing equilibrium solutions - that is, solutions, $A, \rho$, that satisfy $\frac{\partial A}{\partial t} \equiv \frac{\partial \rho}{\partial t} \equiv 0$ - when all are parameters strictly positive. First, we show the existence of a particular homogeneous equilibrium solution in the absence of law enforcement. Indeed, if $\bar{A}, \bar{\rho}$ is a homogeneous equilibrium solution to equation (3.1) then the following equations are satisfied:

$$
\begin{gathered}
0=-\omega \bar{A}+C+\epsilon \bar{\rho} \bar{A} \\
0=\Gamma-\Lambda \bar{\rho}-\epsilon \bar{\rho} \bar{A} .
\end{gathered}
$$

which, formally, yields the two solutions

$$
\begin{aligned}
\bar{A}_{ \pm} & =\frac{\epsilon C+\epsilon \Gamma-\omega \Lambda \pm \sqrt{(\epsilon C+\epsilon \Gamma-\omega \Lambda)^{2}+4 \epsilon \omega C \Lambda}}{2 \epsilon \omega} \\
\bar{\rho}_{\mp} & =\frac{\epsilon C+\epsilon \Gamma+\omega \Lambda \mp \sqrt{(\epsilon C+\epsilon \Gamma+\omega \Lambda)^{2}-4 \epsilon \omega \Gamma \Lambda}}{2 \epsilon \Lambda} .
\end{aligned}
$$




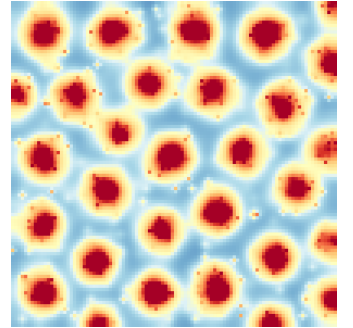

(a) $t=1 \times 10^{3}$

$\mathrm{ACA}=1784$

$B_{\mathrm{Av}}=0.734$

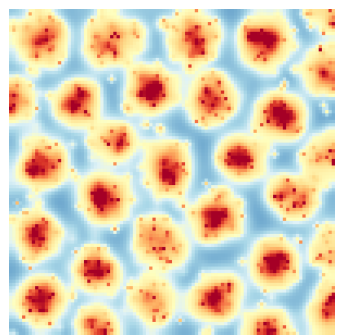

(a) $t=1 \times 10^{3}$

$\mathrm{ACA}=2247$

$B_{\mathrm{Av}}=0.604$

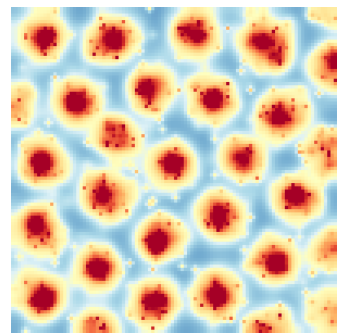

(a) $t=1 \times 10^{3}$

$\mathrm{ACA}=1997$

$B_{\mathrm{Av}}=0.682$

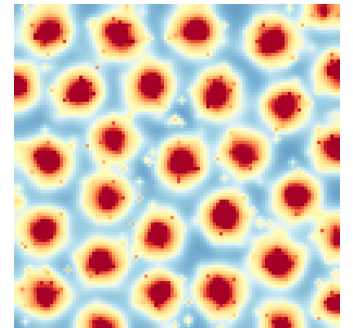

(b) $t=8 \times 10^{3}$

$\mathrm{ACA}=1829$

$B_{\mathrm{Av}}=0.733$

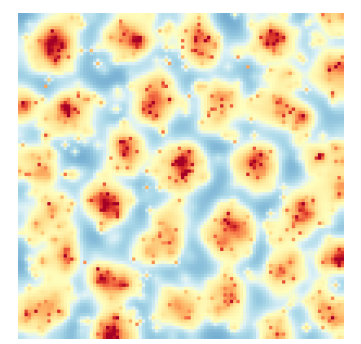

(b) $t=8 \times 10^{3}$

$\mathrm{ACA}=2481$

$B_{\mathrm{Av}}=0.513$

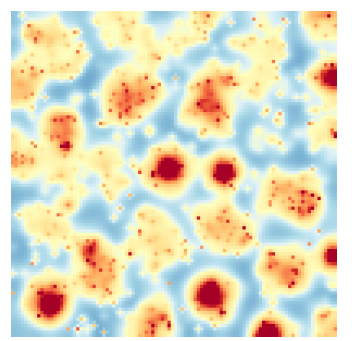

(b) $t=8 \times 10^{3}$

$\mathrm{ACA}=2394$

$B_{\mathrm{Av}}=0.519$

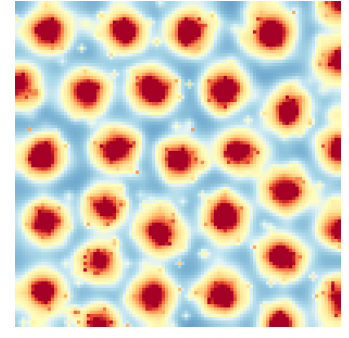

(c) $t=64 \times 10^{3}$

$\mathrm{ACA}=1772$

$B_{\mathrm{Av}}=0.721$

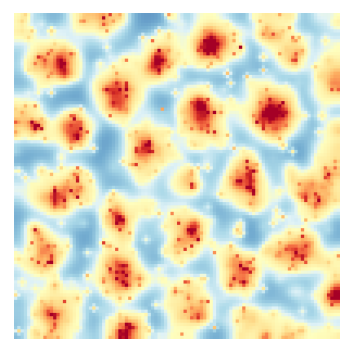

(c) $t=64 \times 10^{3}$

$\mathrm{ACA}=2397$

$B_{\mathrm{Av}}=0.515$

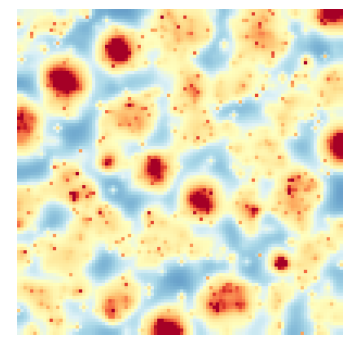

(c) $t=64 \times 10^{3}$

$\mathrm{ACA}=2500$

$B_{\mathrm{Av}}=0.523$
(I) Unbiased Walkers. This method is largely ineffective in criminal activity. Crime hotspots persist despite law enforcement intervention. Only a small reduction in criminal activity, $B_{\mathrm{Av}}\left(64 \times 10^{3}\right)=0.721$, is observed from the initial attractiveness, $B_{\mathrm{Av}}(0)=0.751$.

(II) Cops on the Dots. Criminal activity is reduced. The intensity of crime hotspots has been lowered. There has been a fair reduction in criminal activity, $B_{\mathrm{Av}}\left(64 \times 10^{3}\right)=$ 0.515 a decrease from the initial value of $B_{\mathrm{Av}}(0)=0.751$.

Figure 5: Subfigures I, II, and III compare the relative effectiveness of different law enforcement strategies on a system with pre-existing hotspots. In each case, 300 law enforcement agents are added. To make a law enforcement agent $99 \%$ effective at preventing crime at its current site, $\chi=4.605170$. The simulations are continuations of the simulation displayed in Figure 2 II - that is, the parameters are the same and initial conditions are the state of the system at Figure 2 IIc $\left(t=128 \times 10^{3}\right)$. The unbiased walker patrol method performs the worst. Cops on the dots and peripheral interdiction are roughly comparable after some time, but latter method is less effective for short times. Note that a peculiar side effect of our model is that a reduction in crime is associated with an increase in active criminal agents. This is a result of the fact that fewer criminals committing burglaries implies fewer criminals are being removed from the system. 


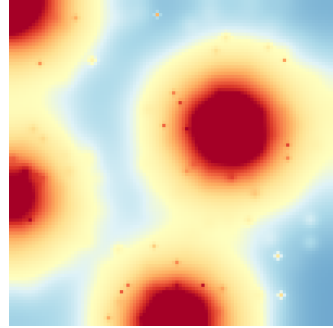

(a) $t=8 \times 10^{3}$

$\mathrm{ACA}=1785$

$B_{\mathrm{Av}}=0.733$

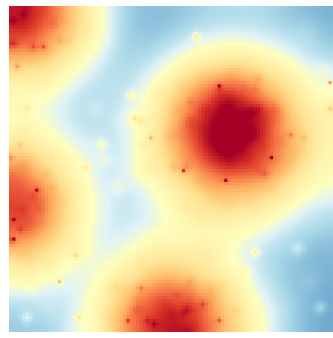

(a) $t=8 \times 10^{3}$

$\mathrm{ACA}=2199$

$B_{\mathrm{Av}}=0.647$

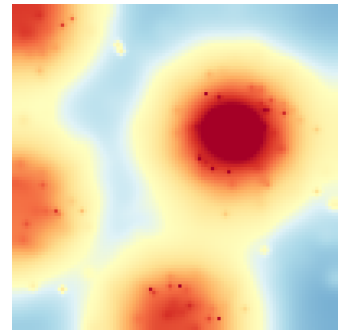

(a) $t=8 \times 10^{3}$

$\mathrm{ACA}=2136$

$B_{\mathrm{Av}}=0.675$

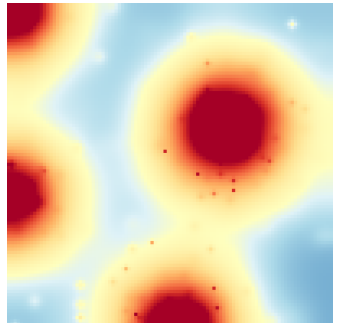

(b) $t=32 \times 10^{3}$

$\mathrm{ACA}=1803$

$B_{\mathrm{Av}}=0.738$

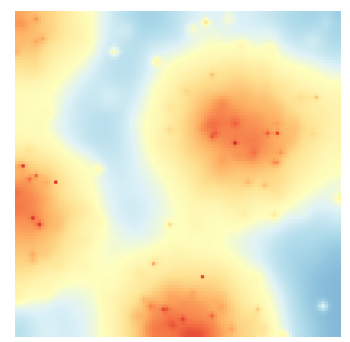

(b) $t=32 \times 10^{3}$

$\mathrm{ACA}=2559$

$B_{\mathrm{Av}}=0.515$

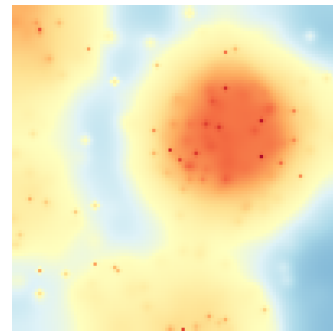

(b) $t=32 \times 10^{3}$

$\mathrm{ACA}=2438$

$B_{\mathrm{Av}}=0.523$

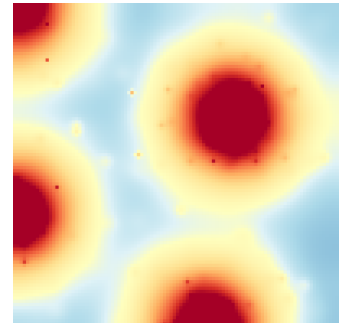

(c) $t=128 \times 10^{3}$

$\mathrm{ACA}=1703$

$B_{\mathrm{Av}}=0.754$

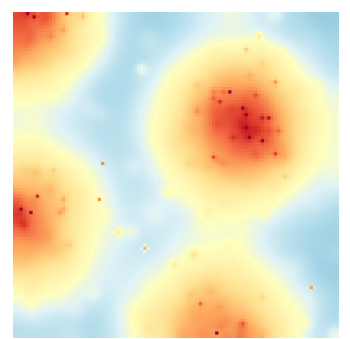

(c) $t=128 \times 10^{3}$

$\mathrm{ACA}=2389$

$B_{\mathrm{Av}}=0.524$

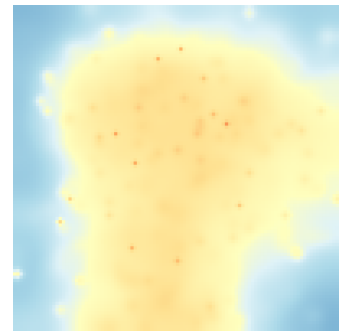

(c) $t=128 \times 10^{3}$

$\mathrm{ACA}=2814$

$B_{\mathrm{Av}}=0.410$
(I) Unbiased Walkers. This method is ineffective in reducing criminal activity. Crime hotspots persist despite law enforcement intervention. There is a slight increase in the attractiveness, $B_{\mathrm{Av}}\left(64 \times 10^{3}\right)=0.754$, is observed from the initial value, $B_{\text {Av }}(0)=0.728$.

(II) Cops on the Dots. Criminal activity is reduced. The intensity of crime hotspots has been lowered. There has been a fair reduction in the attractiveness, $B_{\mathrm{Av}}\left(64 \times 10^{3}\right)=$ 0.524 , a decrease from the initial value of $B_{\mathrm{Av}}(0)=0.728$.

Figure 6: Subfigures I, II, and III compare the relative effectiveness of different law enforcement strategies on a system with pre-existing hotspots. In each case, we add 300 law enforcement agents. We set $\chi=4.605170$; this implies that one law enforcement agent is $99 \%$ effective at preventing crime at its current site. The simulations are continuations of the simulation displayed in Figure 2 III - that is, they share the same parameters and initial conditions as the state of the system at Figure 2 IIIc $\left(t=128 \times 10^{3}\right)$. Again, the unbiased walkers scheme is ineffective. Although cops on the dots performs slightly better for short periods of time, peripheral interdiction is the best method in the long run. 
However since

$$
\epsilon C+\epsilon \Gamma-\omega \Lambda<\sqrt{(\epsilon C+\epsilon \Gamma-\omega \Lambda)^{2}+4 \epsilon \omega C \Lambda},
$$

$\bar{A}_{-}<0$ and hence "aphysical" (i.e., does not correspond to an actual situation). We may therefore eliminate from consideration the pair $\left(A_{-}, \rho_{+}\right)$and have thus proved the following:

Proposition 3.1 If $\kappa(\mathbf{x}, t) \equiv 0$, and $\Gamma, \Lambda, C, \epsilon$, and $\omega$ are positive then there is a unique spatially homogeneous solution of equation (3.1) given by

$$
\begin{aligned}
& \bar{A}=\frac{\epsilon C+\epsilon \Gamma-\omega \Lambda+\sqrt{(\epsilon C+\epsilon \Gamma-\omega \Lambda)^{2}+4 \epsilon \omega C \Lambda}}{2 \epsilon \omega} \\
& \bar{\rho}=\frac{\epsilon C+\epsilon \Gamma+\omega \Lambda-\sqrt{(\epsilon C+\epsilon \Gamma+\omega \Lambda)^{2}-4 \epsilon \omega \Gamma \Lambda}}{2 \epsilon \Lambda} .
\end{aligned}
$$

We are now in a position to examine the effect of law enforcement agents on the equilibrium solution. Suppose that the law enforcement agents are patrolling randomly, that is they are evolving according to equation 2.6.

Eventually, the law enforcement agent density tends to a constant - that is, $\kappa(\mathbf{x}, t)=\kappa_{0}$ - and it is seen that in the system (3.1) we may remove all tildes at the expense of $\epsilon \rightarrow \mathrm{e}^{-\kappa_{0} \chi} \epsilon$. Therefore, with the random strategy, an increase in the number of law enforcement agents is equivalent to a decrease in $\epsilon$. The purpose of adding law enforcement agents to the system was to reduce the amount of crime being committed. Thus, as far as the random strategy is concerned, this amounts to showing that a decrease in $\epsilon$ causes a reduction in crime. First, we must pause to determine how to define "reduction in crime".

In equilibrium, the total attractiveness of the system $\|A\|_{L^{1}}$ is a linearly related to the rate of burglaries as can be seen directly from Eq. 3.2. Moreover, with periodic or Neumann boundary conditions, this is true for long time averages - appropriate limits taken. Indeed, integrating and averaging:

$$
\begin{gathered}
\frac{\partial A}{\partial t}=\eta \Delta A-\omega A+C+\epsilon A \rho=0, \\
\left\langle\|A\|_{L^{1}}\right\rangle=\frac{1}{\omega}\left(C+\left\langle\|\epsilon A \rho\|_{L^{1}}\right\rangle\right) .
\end{gathered}
$$

which is just the equilibrium result. Thus in what follows, we will consider a reduction in $A$ to be equivalent to a reduction in criminal activity.

Since in this section we are concerned with the effect of law enforcement and we have shown the relationship between $\epsilon$ and the equilibrium number of cops, from this point forward we will treat $\epsilon \in \mathbb{R}^{+}$as a parameter. And we will treat various variables as functions of $\epsilon$ when convenient.

The following lemma proves in part a weak version of the statement that an addition of law enforcement officers causes a reduction in the amount of criminal activity.

Lemma 3.2 The equilibrium solution, $\bar{A}$, is an increasing function of $\epsilon$. Likewise, the equilibrium solution, $\bar{\rho}$, is a decreasing function of $\epsilon$. 
Proof. By differentiating equation (3.4) with respect to $\epsilon$ we see that

$$
\begin{aligned}
\frac{\partial \bar{A}}{\partial \epsilon} & =\frac{\Lambda}{2 \epsilon^{2} \sqrt{\left(C+\Gamma-\frac{\omega \Lambda}{\epsilon}\right)^{2}+4 \frac{\omega \Lambda}{\epsilon} C}}\left[\sqrt{\left.\left(C+\Gamma-\frac{\omega \Lambda}{\epsilon}\right)^{2}+4 \frac{\omega \Lambda}{\epsilon} C-C+\Gamma-\frac{\omega \Lambda}{\epsilon}\right]}\right. \\
& >\frac{\Lambda}{2 \epsilon^{2} \sqrt{\left(C+\Gamma-\frac{\omega \Lambda}{\epsilon}\right)^{2}+4 \frac{\omega \Lambda}{\epsilon} C}}\left[\left|C-\Gamma+\frac{\omega \Lambda}{\epsilon}\right|-\left|C-\Gamma+\frac{\omega \Lambda}{\epsilon}\right|\right] \\
& \geq 0 .
\end{aligned}
$$

The first inequality follows from

$$
\begin{aligned}
\left(C+\Gamma-\frac{\omega \Lambda}{\epsilon}\right)^{2}+4 \frac{\omega \Lambda}{\epsilon} C & >\left(C+\Gamma-\frac{\omega \Lambda}{\epsilon}\right)^{2}+4 \frac{\omega \Lambda}{\epsilon} C-4 C \Gamma \\
& =\left(C-\Gamma+\frac{\omega \Lambda}{\epsilon}\right)^{2} .
\end{aligned}
$$

It is noted directly from the combination of Eqs. $3.2-3.3$ that since $\omega \bar{A}+\Lambda \bar{\rho}$ is constant then if one increases the other decreases. Nevertheless, we attack $\rho$ directly:

$$
\begin{aligned}
\frac{\partial \bar{\rho}}{\partial \epsilon} & =-\frac{\omega}{2 \epsilon^{2} \sqrt{\left(C+\Gamma+\frac{\omega \Lambda}{\epsilon}\right)^{2}-4 \frac{\omega \Lambda}{\epsilon}}}\left[\sqrt{\left.\left(C+\Gamma+\frac{\omega \Lambda}{\epsilon}\right)^{2}-4 \frac{\omega \Lambda}{\epsilon}-C+\Gamma-\frac{\omega \Lambda}{\epsilon}\right] .}\right. \\
& <-\frac{\omega}{2 \epsilon^{2} \sqrt{\left(C+\Gamma+\frac{\omega \Lambda}{\epsilon}\right)^{2}-4 \frac{\omega \Lambda}{\epsilon}}}\left[\left|C-\Gamma+\frac{\omega \Lambda}{\epsilon}\right|-\left|C-\Gamma+\frac{\omega \Lambda}{\epsilon}\right|\right] \\
& \leq 0 .
\end{aligned}
$$

Where the first equality follows from

$$
\left(C+\Gamma+\frac{\omega \Lambda}{\epsilon}\right)^{2}-4 \frac{\omega \Lambda}{\epsilon}>\left(C+\Gamma+\frac{\omega \Lambda}{\epsilon}\right)^{2}-4 \frac{\omega \Lambda}{\epsilon}-4 \Gamma C=\left(C-\Gamma+\frac{\omega \Lambda}{\epsilon}\right)^{2}
$$

To obtain a deeper understanding of our system we perform a linear stability analysis at the spatial equilibrium values as described e.g. in [8]. Thus we linearize the system as follows: we perturb the system around the equilibrium values by a constant times $e^{\sigma t+i k \mathbf{x}}$ and study the resulting linear system. We get

$$
\begin{aligned}
\frac{\partial}{\partial t}\left[\begin{array}{c}
\bar{A}+\delta_{A} e^{\sigma t+i k \mathbf{x}} \\
\bar{\rho}+\delta_{\rho} e^{\sigma t+i k \mathbf{x}}
\end{array}\right] & =\sigma e^{\sigma t+i k \mathbf{x}}\left[\begin{array}{c}
\delta_{A} \\
\delta_{\rho}
\end{array}\right] \\
& =e^{\sigma t+i k \mathbf{x}}\left[\begin{array}{cc}
-\eta k^{2}-\omega+\epsilon \bar{\rho} & \epsilon \bar{A} \\
-\epsilon \bar{\rho}+2 \frac{\bar{\rho}}{A} k^{2} & -\Lambda-\epsilon \bar{A}-k^{2}
\end{array}\right]\left[\begin{array}{c}
\delta_{A} \\
\delta_{\rho}
\end{array}\right] .
\end{aligned}
$$


By studying the eigenvalues of the above matrix we can determine when the system is linearly unstable. The two eigenvalues of the system are

$$
\lambda_{ \pm}=\operatorname{Tr} \frac{M}{2} \pm \sqrt{\left(\operatorname{Tr} \frac{M}{2}\right)^{2}-\operatorname{det} M}
$$

where $M$ is the $2 \times 2$ matrix featured in equation $(3.6)$.

$\operatorname{Tr} M<0$ as $-\Lambda-\epsilon \bar{A}-k^{2}<0$ and

$$
-\eta k^{2}-\omega+\epsilon \bar{\rho}=-\eta k^{2}-\frac{C}{A}<0
$$

where the equality in the above display follows from equation 3.2 . Since $\lambda_{-}<0$ the spatial equilibrium is stable - that is $\lambda_{+}>0$ if and only if $\operatorname{det} M<0$. A simple calculation shows that

$$
\operatorname{det} M=\eta k^{4}+\left(\frac{C}{\bar{A}}+\eta \Lambda+\eta \epsilon \bar{A}-2 \epsilon \bar{\rho}\right) k^{2}+\Lambda \frac{C}{\bar{A}}+\epsilon C+\epsilon^{2} \bar{\rho} \bar{A} .
$$

For the time being we will ignore the question of when the spatially homogeneous equilibrium is unstable and only assert that unstable regions exist. Instead we will consider how an unstable system behaves as $\epsilon$ is changed.

Theorem 3.3 Consider fixed constants $\eta, \omega, C, \Gamma, \Lambda>0$. Then there exists constants $\epsilon_{1}, \epsilon_{2}$ so that if $\epsilon<\epsilon_{1}$ or $\epsilon>\epsilon_{2}$ then the solution $\bar{A}(\epsilon), \bar{\rho}(\epsilon)$ is linearly stable.

Proof. To show that a spatially homogeneous solution, $\bar{A}(\epsilon), \bar{\rho}(\epsilon)$, is stable we must prove that $\operatorname{det} M(\epsilon)>0$ for all $k$. Note that the terms which are quartic and constant in $k$ are manifestly positive. Thus it is enough to show that for $\epsilon$ sufficiently small/large the coefficient of $k^{2}$ is also positive. We use the facts:

$$
\lim _{\epsilon \rightarrow 0} \bar{A}(\epsilon)=\frac{C}{\omega} \quad \text { and } \quad \lim _{\epsilon \rightarrow 0} \bar{\rho}(\epsilon)=\frac{\Gamma}{\Lambda}
$$

we can see that

$$
\lim _{\epsilon \rightarrow 0}\left(\frac{C}{\bar{A}}+\eta \Lambda+\eta \epsilon \bar{A}-2 \epsilon \bar{\rho}\right)=(\eta \Lambda+\omega)
$$

Thus by the continuity there exists $\epsilon_{1}>0$, such that the object under the limit is positive for $0<\epsilon<\epsilon_{1}$ which implies that for all $k$, $\operatorname{det} M(\epsilon)>0$. This completes one half of the proof.

Since

$$
\begin{aligned}
\lim _{\epsilon \rightarrow \infty} \epsilon \bar{\rho}(\epsilon) & =\frac{2 \omega \Gamma \Lambda}{C+\Gamma} \quad \text { and } \\
\lim _{\epsilon \rightarrow \infty} \bar{A}(\epsilon) & =\frac{C+\Gamma}{\omega}
\end{aligned}
$$

it is clear that

$$
\lim _{\epsilon \rightarrow \infty} \frac{C}{\bar{A}(\epsilon)}+\eta \Lambda+\eta \epsilon \bar{A}(\epsilon)-2 \epsilon \bar{\rho}(\epsilon)=\infty
$$


Thus there exists $\epsilon_{2}>0$ so that for $\epsilon>\epsilon_{2}$ we have

$$
\frac{C}{\bar{A}(\epsilon)}+\eta \Lambda+\eta \epsilon \bar{A}(\epsilon)-2 \epsilon \bar{\rho}(\epsilon)>0
$$

and thus, for all $k, \operatorname{det} M(\epsilon)>0$ for $\epsilon>\epsilon_{2}$.

Under most conditions, and by most criteria, the presence of law enforcement agents has the desired effect of reducing criminal activity. However with special regards to the $\epsilon>\epsilon_{2}$ portion of the preceding, the preliminary implication is that the addition of law enforcement agents can destabilize a quiescent environment. The statement must be quantified:

1. Although in this regime crime hotspots do emerge with the injection of law enforcement agents - thereby exhibiting increased criminal activity in certain locales - it appears that the overall level of criminal activity actually decreases. Indeed, numerical simulations suggest monotonicity of the total amount of crime with respect to $\epsilon$. Figure 7 clearly shows that $\|A(\cdot, \epsilon)\|_{L^{1}}$ is increasing in $\epsilon$.

2. While no attempt has been in this work to calibrate the simulation dynamics with actual crime statistics, in the authors' opinion the worst (domestic) scenarios roughly correspond to the vicinity of $\epsilon=\epsilon_{1}$. The activity level for $\epsilon \geq \epsilon_{2}$ may correspond to circumstances where drastic alternative modes of response must be considered.

We have seen that, under the full dynamics, cops on the dots tends to outperform the random strategy. By and large, this trend persists with regards to the stability analysis; especially under circumstances of "limited resources".

Theorem 3.4 Let $\bar{A}, \bar{\rho}, \bar{\kappa}$ be a spatially homogeneous equilibrium solution of equations (3.1) and 2.6). If the solution is stable and $\chi \kappa<\frac{\Lambda C}{4 \Gamma \omega}$ then $\bar{A}, \bar{\rho}, \bar{\kappa}$ is a a stable solution of equations (3.1) and (2.7).

We start with a preliminary result:

Lemma 3.5 Suppose $f(x)$ is a monic cubic polynomial satisfying

$$
f(x)=(x+A)\left(x^{2}+B x+C\right) \quad A, B, C>0 .
$$

If $h(x) \equiv f(x)+D A x+\alpha D A^{2}$ where $\alpha>1, D>0$, and $(\alpha-1) D<B$ then $h(x)$ can be written in the form

$$
h(x)=(x+E)\left(x^{2}+F x+G\right) \quad E, F, G>0 .
$$

Proof. Let $g(x) \equiv D A x+\alpha D A^{2}$. First we note that $\lim _{x \rightarrow-\infty} h(x)=-\infty$. As $h(-A)=(\alpha-$ 1) $D A^{2}>0$ we see that $h(x)$ has a root, $x_{0}$, in $[-\infty,-A)$. Let $E=-x_{0}>A>0$. Since $h(0)=$ $E G=A C+\alpha D A^{2}$, we have

$$
G=\frac{h(0)}{E}<\frac{A C+\alpha D A^{2}}{A}=C+\alpha D A<D A+A B+C=h^{\prime}(0)=E F+G .
$$




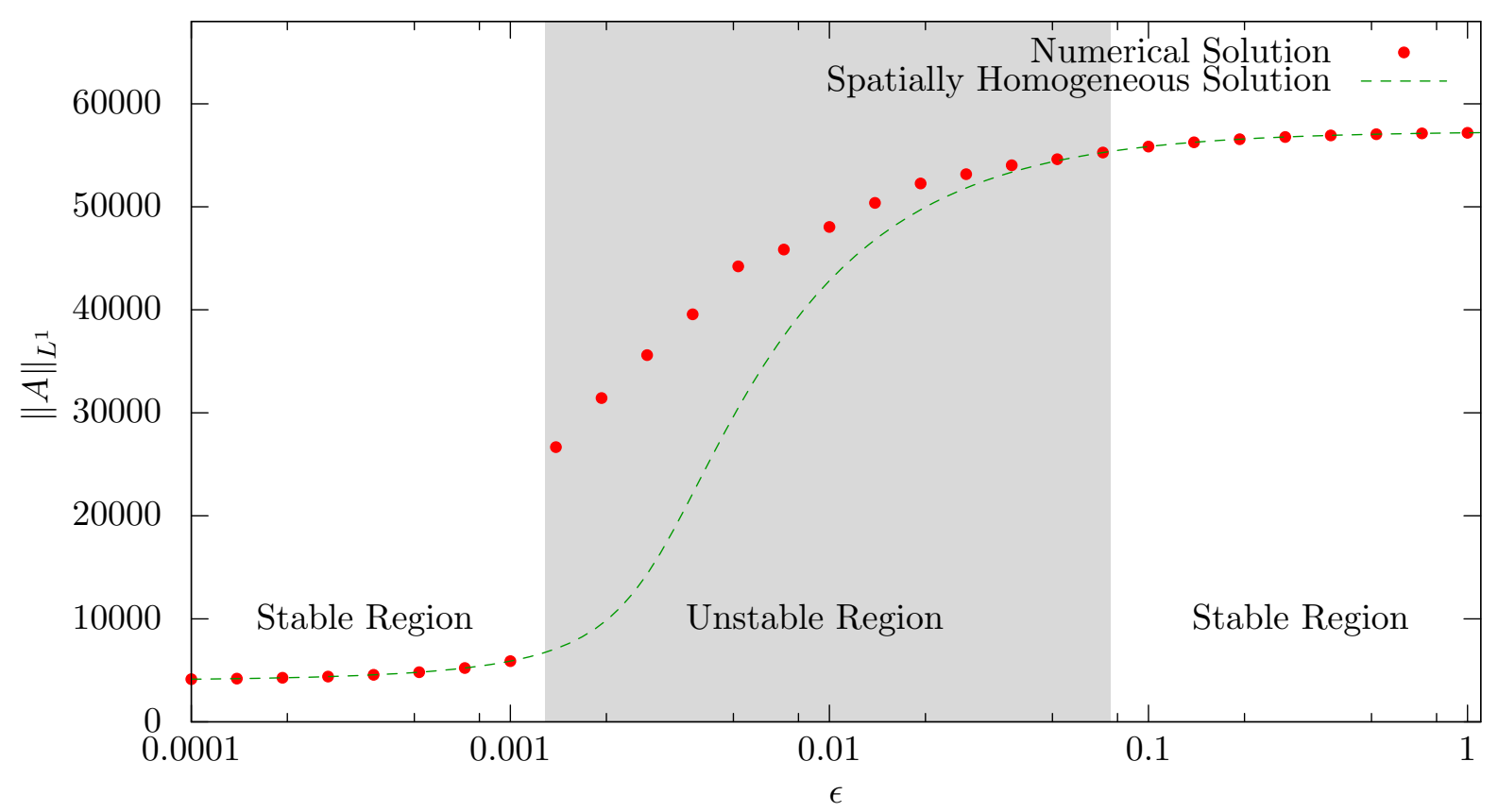

Figure 7: Shows the relationship between the actual and predicted levels of attractiveness. Each numerical simulation was run with parameters $\eta=0.0125, \theta=1.0, \omega=3 \times 10^{-3}, \Gamma=4 \times 10^{-3}$, and $\Lambda=4 \times 10^{-3}$ on $\Omega=[0,200] \times[0,200] \subset$ $\mathbb{R}^{2}$ with Neumann boundary conditions. The system was run to equilibrium. The formation of crime hotspots causes total level of crime to be much larger than the spatially homogeneous level for some values of $\epsilon$.

where the last inequality follows from $(\alpha-1) D<B$. Thus we see that $F>0$. Finally, we note that

$$
G=\frac{h(0)}{E}>0
$$

to complete the proof.

Proof of Theorem 3.4. We begin by perturbing the system around its equilibrium values. We have

$$
\begin{aligned}
& \frac{\partial}{\partial t}\left[\begin{array}{c}
\bar{A}+\delta_{A} e^{\sigma t+i k \mathbf{x}} \\
\bar{\rho}+\delta_{\rho} e^{\sigma t+i k \mathbf{x}} \\
\bar{\kappa}+\delta_{\kappa} e^{\sigma t+i k \mathbf{x}}
\end{array}\right]=\sigma e^{\sigma t+i k \mathbf{x}}\left[\begin{array}{c}
\delta_{A} \\
\delta_{\rho} \\
\delta_{\kappa}
\end{array}\right] \\
& =e^{\sigma t+i k \mathbf{x}}\left[\begin{array}{ccc}
-\eta k^{2}-\omega+\epsilon e^{-\chi \bar{\kappa}} \bar{\rho} & \epsilon e^{-\chi \bar{\kappa}} \bar{A} & -\chi \epsilon \bar{A} e^{-\chi \bar{\kappa}} \bar{\rho} \\
-\epsilon e^{-\chi \bar{\kappa}} \bar{\rho}+2 \frac{\bar{\rho}}{A} k^{2} & -\Lambda-\epsilon e^{-\chi^{\bar{\kappa}} \bar{A}}-k^{2} & \chi \epsilon \bar{A} e^{-\chi \bar{\kappa}} \bar{\rho}-2 \chi \bar{\rho} k^{2} \\
2 \frac{\bar{\kappa}}{A} k^{2} & 0 & -k^{2}
\end{array}\right]\left[\begin{array}{c}
\delta_{A} \\
\delta_{\rho} \\
\delta_{\kappa}
\end{array}\right] .
\end{aligned}
$$

We denote the matrix in (3.11) $N$ and the $2 \times 2$ upper-left sub-matrix of $N$ as $M$. Thus characteristic polynomial of $N, p_{N}(\lambda)$, is

$$
p_{N}(\lambda)=-\left(\lambda+k^{2}\right) p_{M}(\lambda)-2 \chi \bar{\kappa} \epsilon e^{-\chi \bar{\kappa}} \bar{\rho} k^{2}\left(3 k^{2}+\lambda\right) .
$$




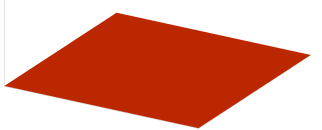

(a) $\epsilon=1.000000 \times 10^{-3}$

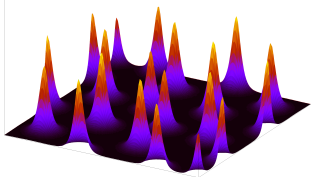

(e) $\epsilon=1.000000 \times 10^{-2}$

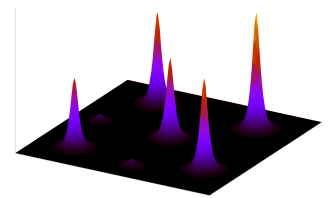

(b) $\epsilon=1.389495 \times 10^{-3}$

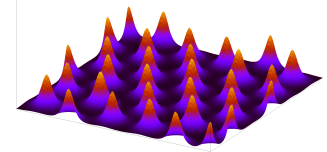

(f) $\epsilon=2.682696 \times 10^{-2}$

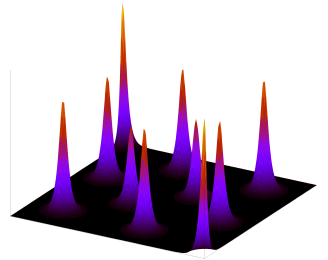

(c) $\epsilon=2.682696 \times 10^{-3}$

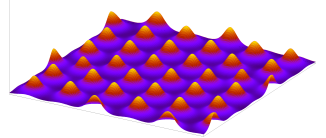

(g) $\epsilon=5.179475 \times 10^{-2}$

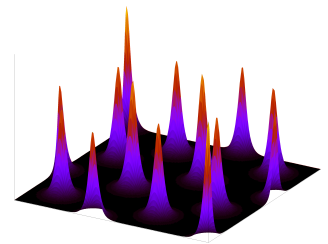

(d) $\epsilon=5.179475 \times 10^{-3}$

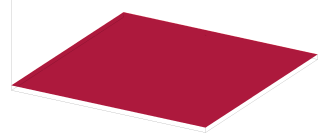

(h) $\epsilon=1.000000 \times 10^{-1}$

Figure 8: Displays the attractiveness, $A$, for some of the numerical simulations shown in Figure 7 Figures $8 \mathrm{a}$ and $8 \mathrm{~h}$ are solutions where the spatially homogeneous solution is stable. Figures $8 \mathrm{~b}-8 \mathrm{~g}$ show solutions where the spatially homogeneous solution is unstable. We note that if $\epsilon$ is small we observe a small number of hotspots that are large in magnitude. If $\epsilon$ is large we observe many hotspots that are small in magnitude.

Now, $p_{M}(\lambda)=\lambda^{2}-\operatorname{Tr} M \lambda+\operatorname{det} M$, where $\operatorname{Tr} M<0$ and $\operatorname{det} M>0$ since we assumed that $\bar{A}, \bar{\rho}, \bar{\kappa}$ is a stable solution of equations (3.1) and 2.6. To show that $\bar{A}, \bar{\rho}, \bar{\kappa}$ is a stable solution of (3.1) and (2.7) we must show that the roots of $p_{M}(\lambda)$ have negative real part. The results follows after an application of Lemma 3.5 which requires that

$$
4 \chi \bar{\kappa} \epsilon e^{-\chi \bar{\kappa}} \bar{\rho}<\epsilon e^{-\chi \bar{\kappa}} \bar{\rho} \frac{\Lambda C}{\Gamma \omega} \leq \epsilon e^{-\chi \bar{\kappa}} \frac{C}{\omega} \leq \epsilon e^{-\chi \bar{\kappa}} \bar{A}<-\operatorname{Tr} M
$$

where the first inequality follows by assumption and the second and third inequalities follow from Lemma 3.2 and the limits in display (3.8).

As it turns out, under more extreme conditions, cops on the dots is not always the most effective strategy for maintaining quiescence. This will be demonstrated after the following:

Lemma 3.6 Suppose $f(x)$ is a monic cubic polynomial satisfying

$$
f(x)=(x+A)\left(x^{2}+B x+C\right)
$$

where $(\alpha-1) A>B>0, C>0$, and $\alpha>1$. Then there exists $D_{0}>0$ so that if $D>D_{0}$ then $h(x) \equiv f(x)+D A x+\alpha D A^{2}$. Then $h(x)$ has a root with positive real part.

Proof. Let

$$
D_{0} \equiv-\frac{f(-A-B)}{(\alpha-1) A^{2}-A B}
$$


Then for $D>D_{0}$ we have

$$
h(-A-B))=f\left((-A-B)+D A^{2}-D A(A+B)>0 .\right.
$$

We also note that

$$
h(-\alpha A)=f(-\alpha A)<0
$$

since the assumption $B<(\alpha-1) A$ implies that any real root of $f(x)$ is greater than $(1-\alpha) A$ as the smallest real root of $x^{2}+B x+C$ is bounded below by $-B$. Thus $h(x)$ has a root, $x_{0}$, satisfying $x_{0} \in(-\alpha A,-A-B)$. Now indeed we can write $h(x)$ in the form

$$
h(x)=\left(x-x_{0}\right)\left(x^{2}+F x+G\right)
$$

for some constants $F, G$. But since

$$
-x_{0}+F=h^{\prime \prime}(0)=f^{\prime \prime}(0)=A+B
$$

we must have

$$
F=x_{0}+A+B<(-A-B)+A+B<0 .
$$

Thus $h(x)$ has a root with positive real part.

With Lemma 3.6 we can show that cops on the dots is not always linearly stable when the random patrol method is linearly stable. If $\eta<1$ we can choose $k^{2}$ such that $-\operatorname{Tr} M<2 k^{2}$. By changing $\kappa, \chi$ and $\epsilon$ we can make the factor in equation 3.12

$$
2 \chi \bar{\kappa} \epsilon e^{-\chi \bar{\kappa}} \bar{\rho}
$$

arbitrarily large while keeping $\epsilon e^{-\chi \bar{\kappa}}$, and thus every other term, constant. Thus we can apply Lemma 3.6 with $D=2 \chi \bar{\kappa} \epsilon e^{-\chi \bar{\kappa}} \bar{\rho}$ to get the result. However, we reemphasize that the circumstances of this "exchanged stability" are not likely to correspond to realistic urban scenarios.

\section{Summary}

In this paper, we have examined the impact of introducing police into models involving mobile criminal offenders and stationary targets, which are known to generate crime hotspot patterns. We studied both discrete agent-based models and related continuum models and find that the introduction of structured policing strategies can eradicate crime hotspots. Specifically, so-called "cops on the dots" is an effective strategy for eliminating smaller hotspots, while so-called "peripheral interdiction" is more effective with larger hotspots. A baseline comparison shows that random patrol does not lead to effective hotspot dissipation. Combined, our results suggest that it may be possible to design spatial policing strategies from first principles. 


\section{Acknowledgments}

This work benefitted immensely from the constructive criticism of Professor Andrea Bertozzi and the advice of Professor Chris Anderson. This research was supported by the NSF under the grant DMS-03-06167 and, for L.C., DMS-08-054856.

\section{References}

[1] L. Anselin, J. Cohen, D. Cook, W. Gorr and G. Tita, Criminal Justice 2000, Vol. 4 (National Institute of Justice, 2000), pp. 213-262.

[2] D. Beavon, P. L. Brantingham and P. J. Brantingham, Crime Prevention Studies, Vol. 2 (Willow Tree Press, 1994), pp. 115-148.

[3] N. Bellomo, A. Bellouquid, J. Nieto and J. J. Soler, Multicellular growing systems: Hyperbolic limits towards macroscopic description, Math. Mod. Meth. Appl. Sci. 17 (2007) 1675-1693.

[4] M. Bendahmame, K. H. Karlsen and J. M. Urbano, On a two-sidely dengenerate chemotaxis model with volume-filling effects, Math. Mod. Meth. Appl. Sci. 17 (2007) 783-804.

[5] W. Bernasco and F. Luykx, Effects of attractiveness, opportunity and accessibility to burglars on residential burglary rates of urban neighborhoods, Brit. J. Criminology 45 (2005) 296-315.

[6] W. Bernasco and P. Nieuwbeerta, How Do Residential Burglars Select Target Areas? The British Journal of Criminology, 45, (2005) 297-315.

[7] A. E. Bottoms and P. Wiles, Crime, Policing and Place: Essays in Environmental Criminology (Routledge, 1992), pp. 11-35.

[8] J. P. Boyd, Chebyshev and Fourier Spectral Methods, 2nd edn. (Dover, 2001).

[9] A. Braga, The Effects of Hot Spots Policing on Crime Annals of the American Academy of Political and Social Science, Vol. 578, What Works in Preventing Crime? Systematic Reviews of Experimental and Quasi-Experimental Research, (2001) 104-125.

[10] P. J. Brantingham and P. L. Brantingham, Patterns in Crime (Macmillan, 1984).

[11] P. J. Brantingham and P. L. Brantingham, Environmental Criminology, 2nd edn. (Waveland Press, 1991), pp. 27-54.

[12] P. J. Brantingham and P. L. Brantingham, Criminality of place: Crime generators and crime attractors, Euro. J. Criminal Policy Res. 3 (1995) 4-26. 
[13] M. Burger, M. Di Francesco and Y. Dolak-Struss, The Keller-Segel model for chemotaxis with prevention of overcrowding: Linear vs. nonlinear diffusion, SIAM J. Math. Anal. 38 (2006) 1288-1315.

[14] H. M. Byrne and M. R. Owen, A new interpretation of the Keller-Segel model based on multiphase modelling, J. Math. Biol. 49 (2004) 604-626.

[15] F. A. Chalub, IY. Dolak-Struss, P. Markowich, D. Oeltz, C. Schmeiser and A. Soref, Model hierarchies for cell aggregation by chemotaxis, Math Mod Meth. Appl. Sci. 16 (2006) 1173.

[16] F. A. Chalub, P. A. Markovich, B. Perthame and C. Scheiser, Kinetic models for chemotaxis and their drift-diffusion limits, Mon. Math. 142 (2004) 123.

[17] L. Cohen and M. Felson, Social-Change And Crime Rate Trends - Routine Activity Approach American Sociological Review 44, (1979) 588-608.

[18] M. C. Cross and P. C. Hohenberg, Pattern formation outside of equilibrium, Rev. Mod. Phys. 65 (1993) 851-1112.

[19] M. del Pino and J. Wei, Collapsing steady states of the Keller-Segel system, Nonlinearity 19 (2006) 661-684.

[20] Y. Dolak and C. Schmeiser, Kinetic models for chemotaxis: Hydrodynamics limits and spatio temporal mechanisms J. Math. Biol. 51 (2005) 595-615.

[21] R. Erban and H. G. Othmer, From individual to collective behavioiur in chemotaxis, SIAM J. Appl. Math 65 (2004) 361-391.

[22] C. Escudero, The fractional Keller-Segel model, Nonlinearity 19 (2006) 2909-2918.

[23] M. K. Felson, Crime and Nature Sage Publications, 2006).

[24] F. Filbet, P. Laurençot and B. Perthame, Derivation of hyperbolic models for chemosensitive movement, J. Math. Biol. 50 (2005) 189-207.

[25] Gottfredson, Michael R., and Travis Hirschi, A General Theory of Crime. Stanford University Press, Stanford, California (1990).

[26] M. A. Herrero and J. J. L. Velázquez, Chemotactic collaspse for the Keller-Segel model, J. Math. Biol. 35 (1996) 177-194.

[27] S. D. Johnson, W. Bernasco, K. J. Bowers, H. Elffers, J. Ratcliffe, G. Rengert and M. Townsley, Space-time patterns of risk: A cross national assessment of residential burglary victimization, J. Quantitative Criminology 23 (2007) 201-219.

[28] S. Johnson, K. Bowers, and A. Hirschfield, New insights into the spatial and temporal distribution of repeat victimisation, The British Journal of Criminology 37, (1997) 224. 
[29] K. Keizer, S. Lindenberg and L. Steg, The Spreading of Disorder Science:1161405, (2008).

[30] E. F. Keller and L. A. Segel, Initiation of slime mold aggregation viewed as an instability, J. Theor. Biol. 26 (1970) 399-415.

[31] G. Kelling, T. Pate, D. Dieckman, and C.E. Brown The Kansas City Preventive Patrol Experiment: A Summary Report Washington, D.C.: Police Foundation, (1974).

[32] M. Lewis, K. White and J. Murray, Analysis of a model for wolf territories, J. Math. Biol. 35 (1997) 749-774.

[33] S. Luckhaus and Y. Sugiyama, Large time behavior of solutions in super-critical cases to degenerate Keller-Segel systems, Math. Model. Numer. Anal. 40 (2006) 597-621.

[34] L. McLaughlin, S. D. Johnson, D. Birks, K. J. Bowers, and K. Pease, Police perceptions of the long and short term spatial distributions of residential burglary, Int. J. Police Sci. Management 9 (2007) 99-111.

[35] P.R. Moorcroft, M.A. Lewis and R. Crabtree, Mechanistic home range models predict patterns of coyote territories in yellowstone, Proc. Roy. Soc. London B 273 (2006) 1651-1659.

[36] H.G. Othmer and T. Hillen, The diffusion limit of transport equations II: Chemotaxis equations, SIAM J. Appl. Math. 62 (2002) 1222-1250.

[37] J. H. Ratcliffe, Crime mapping and the training needs of law enforcement, Eur. J. Criminal Policy Res. 10 (2004) 65-83.

[38] G. F. Rengert, Crime, Policing and Place: Essays in Environmental Criminology, (Routledge, 1992), pp. 109-117.

[39] G. Rengert, Alex Piquero and P. Jones, Distance Decay Reexamined Criminology, 37, (1999) 427-446.

[40] D. Roncek, and R. Bell, Bars, blocks and crime, J. Environ. Sys. 11 (1981) 35-47.

[41] T. Senba, Type II blowup of solutions to a simplified Keller-Segel system in two-dimensional domains, Nonlinear Anal. Th. Meth. Appl. Int. Multidisciplinary J. Ser. A: Th. Meth 66 (2007) 1817-1839.

[42] M. B. Short, M. R. D’Orsogna, V. B. Pasour, G. E. Tita, P. J. Brantingham, A. L. Bertozzi, and L. B. Chayes, A Statistical Model of Criminal Behavior Mathematical Models and Methods in Applied Sciences, 18, Suppl. (2008).

[43] B. Snook, Individual Differences in Distance Travelled by Serial Burglars Journal of Investigative Psychology and Offender Profiling, 1 (2004) 53-66. 
[44] H. Spohn, Large Scale Dynamics of Interacting Particles, Texts and Monographs in Physics, Springer-Verlag, Heidelberg, New York (1991).

[45] A. Stevens, The derivation of chemotaxis equations as limit dynamics of moderately interacting stochastic many-particle systems, SIAM J. Appl. Math. 61 (2002) 183-212.

[46] Y. Sugiyama, Global existence in sub-critical cases and finite blow-up in super-critical cases to degenerate Keller-Segel systems, Differential and Integral Equations Int. J. Th. Appl. 19 (2006) 841-876.

[47] J. J. L. Velázquez, Well-posedness of a model of point dynamics for a limit of the Keller-Segel system, J. Diff. Eqs. 206 (2004) 315-352.

[48] W. F. Walsh, Compstat: An analysis of an emerging police and neighborhood safety, Atlantic Mon. 249 (19822002) 29-38.

[49] D. Weisburd and J. E. Eck, What Can Police Do to Reduce Crime, Disorder, and Fear? The ANNALS of the American Academy of Political and Social Science, 593, (2004) 42-65.

[50] J. Wilson and G. Kelling, Broken windows and police and neighborhood safety, Atlantic Mon. 249, (1982) 29.

[51] R. Wright and S. Decker, Burglars on the Jobs (Northeastern University Press, Boston, 1994).

[52] Federal Bureau of Investigation - Uniform Crime Reports, http://www.fbi.gov/ucr/ucr.htm (Dec 10, 2008). 